\title{
Complexity in Pharmaceutical Powders for Inhalation: A perspective ${ }^{\dagger}$
}

\author{
Anthony J. Hickey \\ ${ }^{1}$ RTI International, USA
}

\begin{abstract}
Pharmaceutical aerosol powders intended for inhalation are required to have unique performance characteristics that are highly dependent on their physico-chemical properties. A wide range of analytical methods have been applied to study particle structure, size distribution, surface properties and subsequent behaviour of powders with the intention of predicting their performance. However, particle interactions are dictated by complex fundamental forces that impact on the efficiency and reproducibility of delivery and thereby the quality, efficacy and safety of the final product. This manuscript reviews the interplay of physico-chemical properties of powder and the complex process and analytical variables that must be monitored and controlled to effectively predict powder performance.
\end{abstract}

Keywords: powder, particle, drug, inhalation, characterization

\section{Introduction}

Understanding the role of powder properties in their performance can be likened to the aphorism of the blind men and the elephant (Shah I., 1993). All methods, applied correctly, describe a specific feature of the particle or powder. However, without knowledge of all of the features that define the whole object or population of objects the conclusions about it/them may be misleading. The challenge to the pharmaceutical research and development community has been to collect sufficient information on powder properties to allow prediction of behavior.

Interest in powder properties with respect to ensuring efficient and reproducible performance in delivering drugs from inhalers has driven an enormous research effort over a period of more than 50 years. The complex interaction of fundamental aspects of physical chemistry, mechanical engineering, aerosol physics and the ability to identify and consider all relevant variables has limited the development of comprehensive predictive models of product behavior (Hickey A., 2003). The current capacity to use the knowledge of key parameters to guide product optimization appears to involve systematic consideration of the most important factors but requires iteration with respect to unidentified variables or confounding of known

Received 21 December 2016; Accepted 15 February 2017

J-STAGE Advance published online 8 April 2017

3040 Cornwallis Road, Research Triangle Park, NC 27709, USA

E-mail: ahickey@unc.edu

TEL: +1-919-541-6771 FAX: +1-919-541-6936 variables that lead to sub-optimal performance (Hickey A. and Xu Z., 2013).

It is important to recognize that dry powders are non-equilibrium systems (although time scales can be long) at all dimensional scales of scrutiny. The historical view of individual particles as essentially unchanging objects has to be revised to reflect their ability to respond to manufacturing, storage and general environmental conditions. This has implications for their interaction with each other and the container or conveyor which influences bulk powder properties.

The intent of this review is to describe the factors known to contribute to dry powder inhaler product performance and then to discuss approaches to characterization that might account for interactions of these variables or for unknown variables to contribution.

\section{Methods of manufacture and particle morphology}

\subsection{Milling}

Milling has a history that can be traced through centuries for the processing of raw materials for food and pharmaceutical products. The desired particles size for aerosol delivery of drugs to the lungs requires a high-energy method to overcome the forces of attraction between molecules usually in the crystalline solid state to create a large surface area (Hickey A. and Ganderton D., 2001; Shur J. et al., 2012). The method, as depicted in Fig. 1, 
(a)

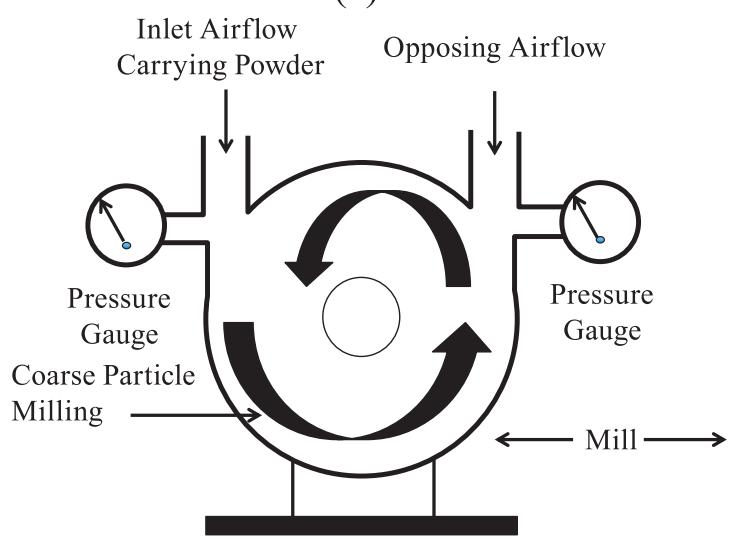

(b)

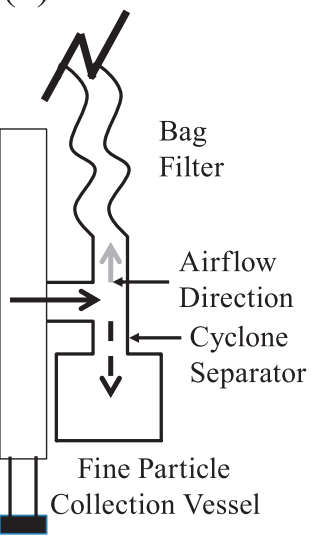

Fig. 1 Schematics of (a) front view of an air jet mill and (b) side view showing the filter and collection vessel separated by the cyclone behind the mill.

employed throughout modern times is air jet milling in which particles are carried on high a velocity gas stream (usually air or nitrogen) into an opposing high velocity gas stream such that particles impinge on each other and the walls of the mill shattering ultimately into respirable sizes $(1-5 \mu \mathrm{m})($ Fig. 1a). These respirable particles are collected by elutriation, in the figure passing into the plane of the image through a central tube and entering a cyclone separator as shown in Fig. 1b (Hickey A., 1993). In the example shown air then passes to a bag filter and exits while powder under the influence of impaction and sedimentation forces is collected in a vessel directly below the filter. This process can be continued for as long as necessary to the capacity of the collection vessel and with attention to occlusion of the filter.

The production of particles by milling can be controlled in terms of the processing variables of gas flow and temperature but cannot readily be controlled at the level of the individual particle production and the outcome of the process is highly dependent on the starting material in terms of both composition and state which makes it a difficult process from which to extract general principles. Each drug treated in this manner requires full characterization in order to guarantee quality and performance in the final product.

\subsection{Spray drying}

Spray drying has been of increasing interest since over the last 20 years as it became evident that the technique resulted in particles and powders with unique physicochemical and performance properties (Vehring R., 2008; Van Oort M. and Sacchetti M., 2007). The ability to create low-density particles promoted the ability to efficiently disperse particles. Since most spray dried material is amorphous in nature the influence on stability needs to be addressed. For proteins and peptides this is a desirable

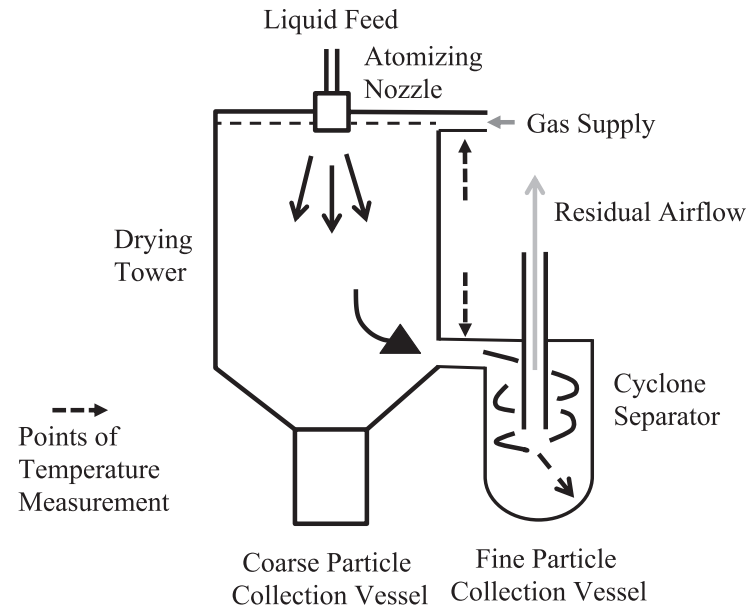

Fig. 2 Schematic of a spray drier illustrating the key elements of solution feed, gas supply, temperature control and monitoring and collection vessels before and after the size selective cyclone separator.

solid-state form and is likely to be stable on storage. For small molecular weight drugs the amorphous form is unlikely to be the most stable with respect to fluctuations in moisture and temperature (Carstensen J., 1990). Consequently, since most drugs are of small molecular weight, packaging considerations are required to ensure the stability of the final product most notably the use of moisture impermeable wrapping, e.g. aluminum, or inclusion of desiccant where the response to equilibrium moisture content and fluctuations are more modest (Atkins P. and Crowder T., 2004).

Fig. 2 illustrates the key components of a spray drier including the liquid feed to the atomization nozzle, drying gas supply, the drying tower, cyclone separator and collection vessels for coarse and fine particles. This is a simplified image as it does not show condensers, heater/ cooler, recycling and feedback electronics that are integral parts of this equipment. 
203 Supercritical fluid manufacture

Supercritical fluid manufacture showed great promise as a means of accurately and reproducibly controlling particle size and morphology when it first appeared as an approach to the manufacture of drug particles in the 1990s (Van Oort M. and Sacchetti M., 2007). Several methods were developed each of which had its merits but all of which required multiple small batch production cycles to achieve pilot or production scale quantities of processed drug. Rapid expansion supercritical solutions or antisolvent techniques are the most prominent methods using supercritical gases such as carbon dioxide.

\subsection{Mixing/Blending}

The modern history of dry powder inhalers can be traced back to the 1960s in particular to the Cromolyn Sodium product delivered from a Spinhaler (Pfisons) (Sanders M., 2011). From the outset mixing/blending was a central element of the powder formulation and the concept of attaching milled respirable particles to the surface of lactose carrier particles became the standard, which remains most common to this day. The importance of the mixing process cannot be understated as it dictates both the ability to fill the dosing containment unit (e.g. capsule, blister or reservoir) and the efficiency with which the drug particles can be detached and enter the entraining air supply (Stewart P., 1986; Young P. et al., 2007).

\section{Physico-chemical properties}

\subsection{Primary particle size and distribution}

Primary particle size and distribution is an important property to measure for aerosol products. The aerody- namic performance of aerosols depends on the ability to input energy usually from the patient's inspiratory airflow to move the powder and disperse it into particles that can enter the lungs. This non-equilibrium phenomenon rarely disperses drug completely into primary particle sizes. However, by measuring the primary particle size and distribution an important metric for underlying quality as well as a target for complete dispersion is established. Primary particle size can be evaluated by two complementary methods an indirect population measurement. Laser diffraction measurement can be conducted to assess particles in liquid suspension or dry powders at highpressure drop, to allow sufficient shear to break the powders in individual particles (Behara S. et al., 2012; Jaffari S. et al., 2013; 2014). Scanning electron microscopy allows imaging on a scale to visualize particles in respirable size ranges (Hickey A. and Xu Z., 2013). Individual particles can be distinguished from aggregates. However, microscopy generally does not allow sufficient numbers of particles to be measured for quantitative statistical representation of the population of particles (Allen T., 1990).

\subsection{Shape and density}

Particle shape is an important property dictating their behavior. Fig. 3 illustrates schematically important features of particle surfaces. While the ideal particle is spherical (Fig. 3a(i)) and is the basis for most measures of particle size (equivalent spheres) most drug particles deviate from sphericity and exhibit some form of surface rugosity (Fig. 3a(ii)) (Chew N. and Chan H-K., 2001). In the extreme the shape of the particle can be significantly distorted in one (needle or fiber, Fig. 3a(iii)) or two (plate or flake) dimensions (Mullin J., 1993). The latter usually occurs because of restriction of crystal growth in one (plate or flake) or two (needle or fiber) dimensions. The shape of the particle and surface functionality are inextricably

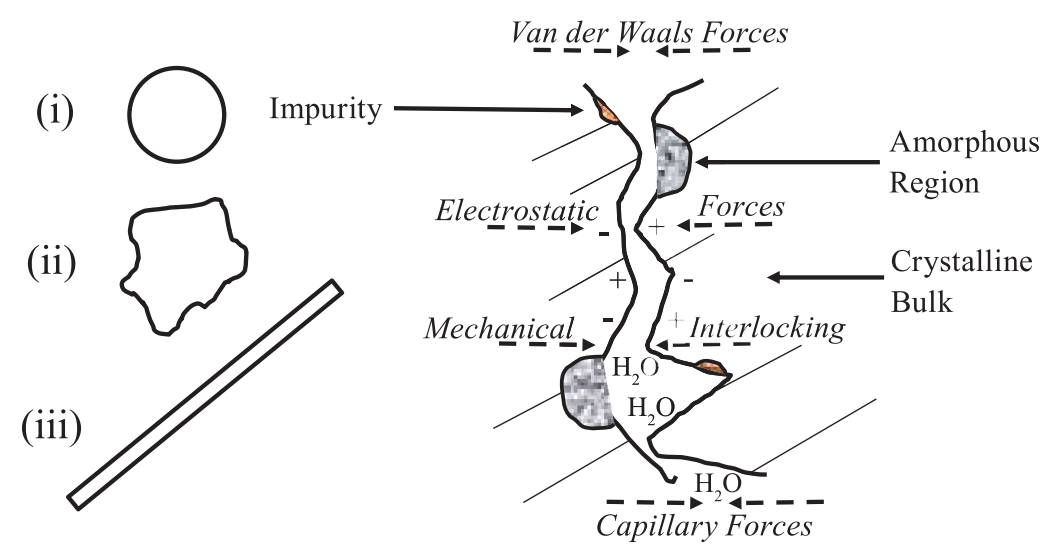

Fig. 3 Schematics illustrating (a) (i) spherical, (ii) rugose or (iii) elongated Particle morphology and (b) Forces of interaction at the interface between two particle surfaces. 
limkedksince the surface may exhibit specific features based on the action of impurities during crystallization or the presence of different polymorphs or moisture at the surface. Fig. 3b illustrates a variety of surface phenomena resulting from the presence of moisture, crystallinity, electrostatics, discontinuities and, impurities. These surface functions increase the interaction of particles beyond that resulting from the innate van der Waals forces.

Once features exist the way in which they border three-dimensional space with edges or corners creates both a physical and energetic differential that impacts on the likelihood of interaction with other surfaces.

Some mathematical approaches have been taken to depicting irregularly shaped particles with a view to correlating the numerical descriptor with subsequent behavior. Fig. 4 shows two methods that have been applied. The first is to inscribe the image of the particle periphery with radii from a central point that can then be described in polar co-ordinates, distance from the origin for designated angles, from which Fourier coefficients can be derived that are correlated with a variety of shapes (Tian Y. et al., 1994; Hickey A. and Concessio N., 1997). First, second, third, etc. Fourier coefficients are considered to represent roundness, squareness, triangularity, and so on.

Fractal analysis has been applied to the shape of particles as a means of expanding shape considerations beyond conventional simple shape factors more descriptive terms such as Fourier coefficients based on polar co-ordinates (Hickey A. and Concessio N., 1997). The Fractal dimension introduced for the first time the notion of non-integer dimensions $<3 D$ to convey greater information about the particle than the usual one or two dimensional descriptors obtained from imaging individual particles and subse-

(a)
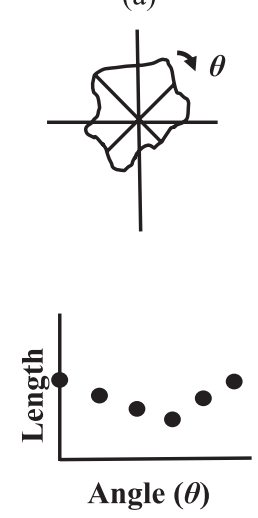

Fig. 4 Depiction of (a) Polar co-ordinate image and the data derived for analysis that would be fit to a Fourier series to obtain coefficients and (b) The use of different scales, top, to measure the surface by connecting chords around the periphery, middle, to allow estimates of the perimeter length to be plotted against the stride length on a log-log scale, bottom, from which the Fractal Dimension, $\delta$, is measured. quently statistically aggregated into particle population data represented by the number of particles observed and then described in terms of average and distribution.

The aerodynamic behavior of aerosol particles is dictated by the particle size and other physical properties including shape and density as described by Stokes' equation. The importance of these properties was overlooked until relatively recently when the potential to modify them to enhance aerosol performance was realized (Crowder T. et al., 2002).

Spray drying may be employed to produce low-density particles. The method involves the spraying of hollow shell or highly porous particles (whiffle ball) that present delicate membrane-like surfaces in various geometric structures permeated by air (Vehring R., 2008; Van Oort M. and Sacchetti M., 2007). These particles due to their low density (somewhere between the true density of a solid particle and the density of air) may be quite large but have a small aerodynamic diameter as described by Stokes' Law. In addition, the distance between the molecules of drug in the particle and those in adjacent particles means that the van der Waals forces are much lower than a solid particle due to the reduced Lennard Jones potential (Telko M. and Hickey A., 2005; Dunbar C. et al., 1998). Consequently, these particles require very little energy to disperse as an aerosol. Indeed, if placed on a flat surface in room air the local convective air movement may be sufficient to generate the aerosol.

Elongated particles are known to orientate longitudinally into the direction of the airflow (Davies C., 1979; Gonda I. and el Khalik A., 1988; Fults K. et al., 1997). Consequently, for specific length, cross-sectional area diameter and aspect ratio they have the capacity to move in air predominantly as a function of their cross-sectional area diameter suggesting a limited radius of gyration while in motion in the two dimensions represented by the cross section (Lippmann M., 1990).

Most dry powder inhaler products employ blends of drug with lactose. The surface properties of the drug and the lactose play a significant role in their interaction and, thereby, in the energy required to separate and disperse them into primary drug and lactose particles $(\mathrm{Xu} \mathrm{Z}$. et al. 2011). The use of $\propto$-lactose monohydrate a monoclinic crystal presents proportionately large flat surfaces to the smaller drug particles (Hickey A. et al., 2007a and 2007b). These surfaces are deceptive in their apparent uniformity in that they can become discontinuous through the presence of impurities (Crowder T. et al., 2003). The presence of impurities, moisture, amorphous lactose or $\beta$-lactose monohydrate at the surface creates variable surface energy densities for interaction with the drug (Fig. 3b) (Hickey A. et al., 2007a). The potential for variation in nature and extent of drug binding gives rise to non-uniformity in performance, which has implications 
for both quality and therapy (Telko M. and Hickey A., 2005; Dunbar C. et al., 1998).

A variety of methods of analysis have been evaluated for their utility in characterizing surface forces including thermal analysis (bulk polymorphic forms) inverse gas chromatography (surface polar and non-polar forces, (Telko M. and Hickey A., 2007)), atomic force microscopy (surface free energy, (Begat P. et al., 2004)), static (Carstensen J., 1993) and dynamic (Hickey A. and Concessio N., 1996) angle of repose (bulk flow properties as a measure of particle interaction), Faraday cage (standing electrostatic charge, (Smyth H. et al., 2004)), electronic samplers for charge determination as a function of aerodynamic particle size (Telko M. et al. 2007; Telko M. and Hickey A., 2014; Wong J. et al., 2015).

\section{Complexity}

The ability to predict powder performance has clear implications for ensuring the quality and efficacy of a pharmaceutical aerosol formulation. In addition, accurate prediction would allow efficient and cost-effective product development with a high probability of meeting with regulatory approval by accommodating the desire for quality by design.

It would be scientifically elegant to approach the desire to predict powder performance from the perspective of the fundamental underlying forces of interaction between particles discussed earlier. However, the inability to separate these forces in the heterogeneous systems of which pharmaceutical formulations are composed has confounded efforts to reduce the problem to basic principles. Various groups have chosen to examine the problem from the perspective of probing powder systems experimentally to derive data on performance that can inform models that allow prediction of behavior (Hickey A. and $\mathrm{Xu}$ Z., 2013; Steckel H. and Bolzen N., 2004; Jones M. and Price R., 2006; Grasmeijer F. et al., 2013, 2014).

The powders employed for inhalation consist predominantly of blends with lactose or as spray-dried particles. It is important in considering the dispersion properties of a powder to consider it as a function of quantifiable descriptors of the energy imparted by the airflow such as Reynolds' number, resistance or shear stress. These terms can easily be described for most devices but it should be noted that all devices include design features intended to aid in deaggregation such as baffles, tortuous channels or other impaction surfaces (Telko M. and Hickey A., 2005). Consequently, when considering terms such as pressure drop (at a fixed airflow rate) or airflow rate (at the defined device pressure drop) across or through the device as a reference term for the energy imparted the design features may make the data deviate from quantitative generalizable relationships while giving qualitative information about formulation performance.

Fig. 5 illustrates a model of the proposed interactions between drug and lactose assuming regions of different surface energy density exist to which drug particles exhibit a range of binding properties. When subject to shear and turbulence in air, in the mouthpiece or when passing through or around a tortuous air path feature, impacting on device surfaces and with other particles drug is liberated from the surface of lactose by the action of airflow and constraints of the air path. Incomplete dispersion may be explained by the heterogeneity of the surface energy density.

Fig. 6 illustrates a second model proposed for interactions between drug and lactose assuming that not only is drug associated directly with lactose but through cohesion and incomplete mixing aggregates exist at the surface of lactose that because of their larger size are stripped more readily from the surface and then deaggregated by the shear, turbulence and impaction described earlier. This model is not mutually exclusive with respect to the first model but adds a layer of additional information that might help in formulation that being the cohesive or adhesive nature of the component particles.

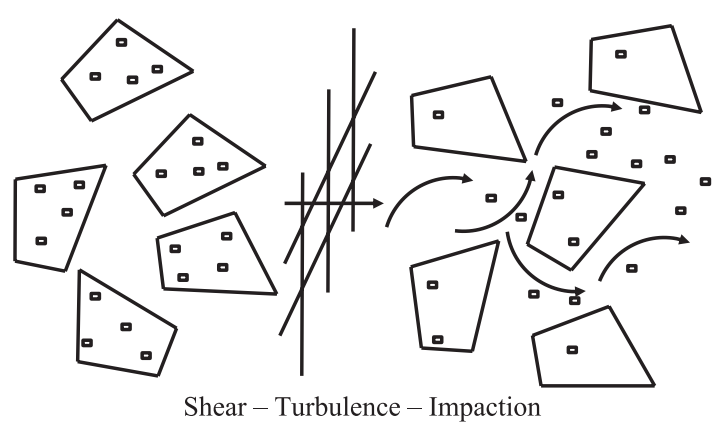

Fig. 5 Schematic of small drug particles at the surface of large lactose carrier particle depicting their liberation as a function of airflow and air path features.

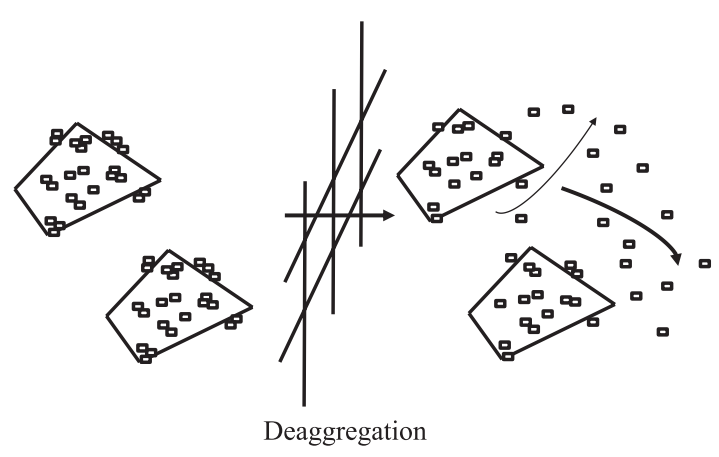

Fig. 6 Schematic of small drug particles at the surface of large lactose carrier particles some in the form of aggregates depicting their liberation initially as a deaggregation phenomenon in response to airflow. 


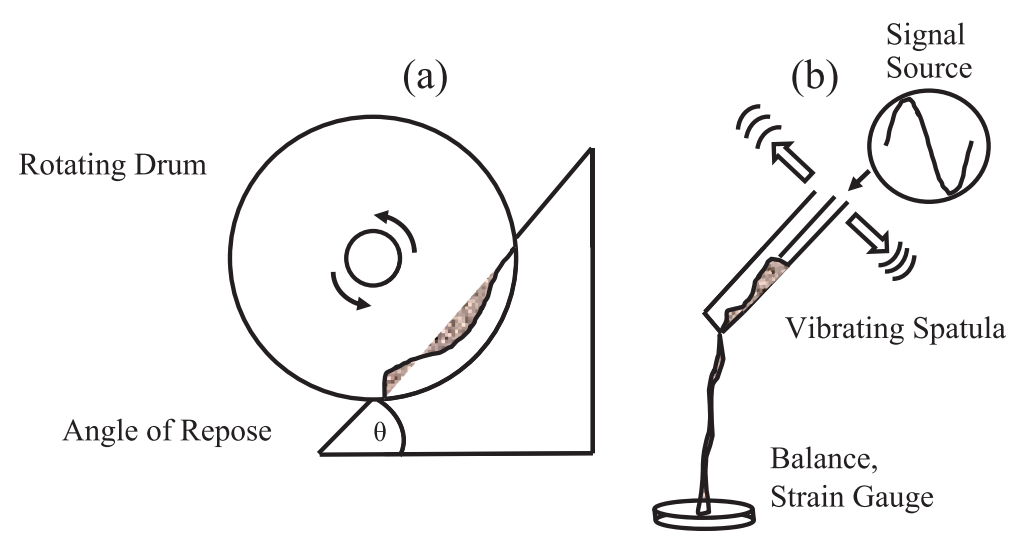

Fig. 7 Diagrams of (a) the rotating drum dynamic angle of repose measurement technique and (b) vibrating spatula with mass detection.

\subsection{Powder flow}

The movement of most pharmaceutical powders is irregular but almost all processes in which powders play a role would be better served by uniform flow and behavior including aerosol generation. Since the nature of powder movement can vary it can be inferred that knowledge of this phenomenon would be useful in predicting a variety of powder properties such as dilation followed by generation for aerosol formulations.

There are several methods for evaluating powder flow properties and their underlying physics including rotating drum (Hickey A. and Concessio N., 1996; Crowder T. et al., 1999, Crowder, T. and Hickey A., 1999), vibrating spatula (Hickey A. and Concessio N., 1994; Crowder T. and Hickey A., 1999) and critical orifice (Flodex, (Taylor M. et al., 2000)). When oscillations in the motion of powder in a rotating drum or delivery from a vibrating spatula, as depicted in Fig. 7, are plotted in phase space a typical attractor is observed that can be characterized by a Fractal Dimension $(\delta)$ or Lyupanov Exponent reflecting the divergence of each successive data point from the previous one. This single dimension, which can be viewed as a measure of irregularity in microflow can then be correlated with the ability to disperse powders as aerosols (Concessio N. et al., 1999; Crowder T. and Hickey A., 2006). The predictive nature of this approach renders it a potentially useful tool for the characterization of powder blends. Formulation optimization might be performed by ranking powders in terms of flow (powders with small $\delta$ flow better than those with large $\delta$ ) as a predictor of their ability to effectively be disperse as aerosols.

\subsection{Cohesive adhesive balance}

The cohesive adhesive balance (CAB) model refers to the interaction between drug and lactose in the blend that influence the energy required for detachment of particles from each other and surfaces during the aerosolization process (Begat P. et al., 2004; Behara S. et al., 2011a; $2011 b ; 2011 c)$. Drug entrainment onto the inspiratory flow of the patient is influenced by these interactions and consequently may result in variable efficacy (Grasmeijer F. et al., 2013; Behara S. et al., 2011d).

\subsection{Powder aerosol dispersion equation}

Collecting experimental data and developing a working model, rather than a physical model might circumvent the physical complexity of the models of interaction between particles in a blend. One approach to measuring the interaction of particles and data collection is to investigate the energy required to separate them in a standardized airflow using an entrainment tube (Fig. 8a) and inertial impactor for aerodynamic particle size measurement (Fig. 8b). This approach has been explored for several drugs in lactose blends (Mansour H. et al., 2010; Xu Z. et al., 2010a, $2010 \mathrm{~b}, 2010 \mathrm{c})$. The detachment in response to applied shear stress was considered analogous to desorption phenomena in which particles are removed from low energy binding sites and increasing quantities are removed at high shear as higher energy binding sites are evacuated (Xu Z. et al., 2010c). These experiments resulted in the proposal of a powder aerosol dispersion equation (PADE) (Xu Z. et al., 2010c).

(a)

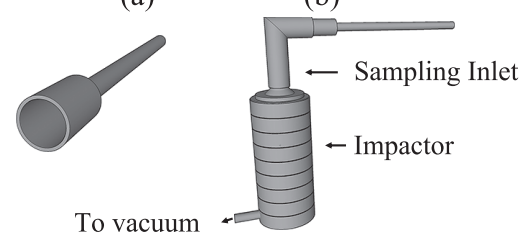

Fig. 8 Illustrations of (a) a standardized entrainment tube and; (b) its position with respect to a stacked, cascade impactor for aerodynamic particle size determination. 


\subsection{Aerosol delivery rate}

\subsection{Laser diffraction volume diameter determination}

Laser diffraction instruments are used routinely for measurement of particle size distribution of aerosol powders. When used with powder dispersion systems operating at different pressure drops or when dispersed in suspending liquid particles may be separated into primary sizes to establish the limit of efficient dispersion possible with an effective dry powder inhaler (Young P. et al., 2007; Behara S. et al., 2012). The particle size obtained at different dispersing pressure drops may be used to characterize the ease of deaggregation (resistance to deaggregation due to particle interactions).

\subsection{Aerodynamic particle size distribution}

The aerodynamic particle size distribution (APSD) is generally measured by inertial impaction (Tougas $\mathrm{T}$. et al., 2013). Inertial impactors size segregate powders using a series of jets of decreasing orifice size through which air is drawn, at a fixed rate by a vacuum. An increasing linear velocity at each successive collection stage is thereby established. The combination of the mass of particles in the distribution and range of linear velocities results in samples of decreasing particle size being sampled as an aerosol passes through each successive stage of the impactor.

\subsection{Delivered dose}

The dose delivered from the inhaler is that which leaves the mouthpiece of the device for which a proportion, the fine particle dose, is considered in a size that is relevant to pulmonary delivery (USP, 2011). The fine particle dose should not be confused with the respirable dose. The fine particle dose is designated as the entire dose below a specific particle size, usually $5 \mathrm{~mm}$ unless otherwise stated, that is thought to be broadly relevant for pulmonary drugs as a quality measure (Hickey A., 2004). The respirable dose is derived from theoretical and experimental data on lung deposition and considers the probability of particles over a range of sizes to penetrate to the lungs. Various public and occupational health agencies have specific definitions for respirable dose (Hinds W., 1999). The fact that a single fine particle doses is equivalent to a range of respirable doses based on the particle size distribution has been considered elsewhere (Martonen T. et al., 1992) It is important to note that caution must be exercised in interpreting fine particle dose, a quality measure, as a predictor of lung delivery.
In evaluating the performance of DPIs the importance of aerosol delivery rate (ADR) is often overlooked. The APSD and delivered dose uniformity (DDU) are clearly important quality measures for the product performance but the relevance of ADR may not be as evident. The relevance of ADR only becomes obvious when the purpose of a DPI is considered. Drug delivered as an aerosol from a DPI is intended, within reasonable limits, to be controlled with respect to the characteristics that may be important in lung delivery and, therefore, in therapeutic effect. The portion of the inspiratory flow on which the aerosol is delivered is critical to its site of deposition and the ADR, or mass of aerosol powder delivered at different times with respect to initiation of airflow is an important indicator of performance in patients (Ziffels S. et al., 2015). Fig. 9 illustrates this point by adopting an approach of following obscuration of a light source placed perpendicularly to the direction of aerosol delivery as sampling into an inertial impactor is performed (Fig. 9a). For illustration the aerosol dispersion is separated into three periods initially visualizing increasing mass, followed by peak mass and terminating in declining mass delivery (Fig. 9b). This is measured by a detector in which obscuration is the reciprocal of light detected. Differences in the shape of the obscuration curve indicate differences in ADR (Fig. 9c).

The complexity of dry powder inhalers can only be fully grasped when the need for control at all scales spatial and temporal scrutiny is considered. Fig. 10 summarizes the dimensions, the properties, the processes and the control variables that can be employed to influence each of the systems. There are points in the preparation of dry powders for inhalation where certain properties are known to positively influence the aerosol performance of the powder. Arguably, great progress has been made with regard to qualitative understanding of which factors may be important in supporting product development and by iteration performance can be optimized sufficiently to meet regulatory requirements.

Particle surface treatments such as smoothing (Young P. et al., 2002), mechanical coating (Zhou Q. et al., 2013), corrugation (Chew N. and Chan K-K., 2001) and the use of fines to obscure high surface energy density sites (Jones M. and Price R., 2006) are among the promising strategies to limit the influence of formulation variables on performance. The use of standardized techniques to evaluate the formulation independently of the device allows initial optimization (Louey M. et al., 2006) and the use of inspiratory flow profiles in sampling may be more sensitive to performance variation than constant flow rates currently used (Dunbar C. et al., 2000). Ultimately, the device and formulation combination must be evaluated (Behara S. et al., 2011d; Adams W. et al., 2012) to 
(a)

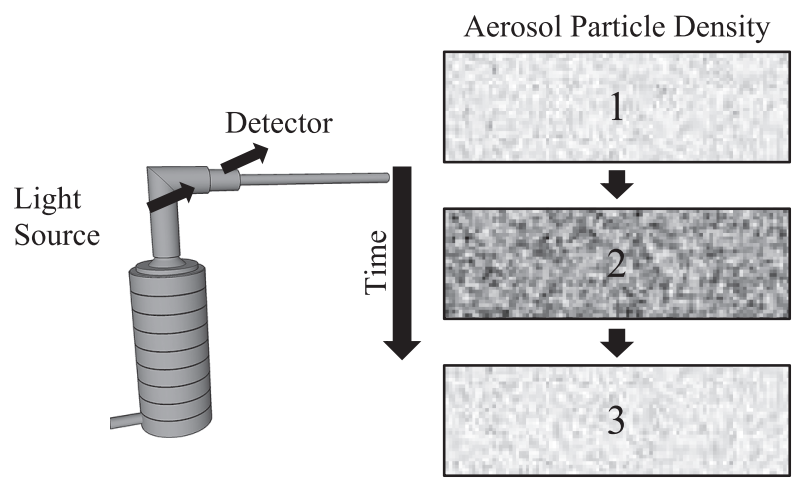

(c)

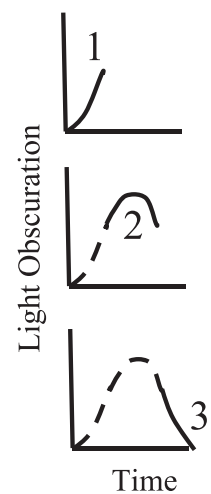

Fig. 9 (a) Arrangement of apparatus to follow light obscuration at the inlet to an inertial impactor; (b) Depiction of the density of particles in air (dictating light obscuration) at three intervals after (top to bottom) initiation of aerosol delivery during aerosol delivery; (c) The real-time measurement of obscuration at the detector corresponding with (b), initial, middle and end (top to bottom).

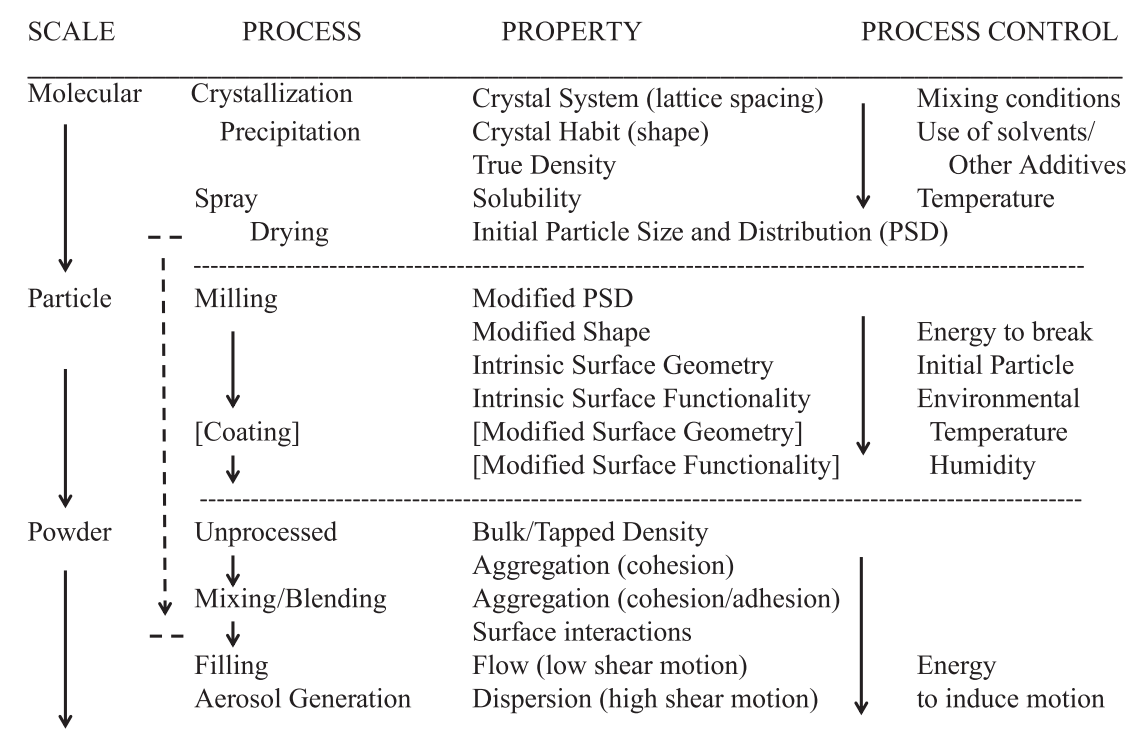

\section{DESIRED PERFORMANCE CHARACTERISTICS}

Fig. 10 Properties with respect to scale of scrutiny of a pharmaceutical dry powder aerosol formulation and the processes and variables that must be controlled to optimize desired performance characteristics with respect to quality, safety and efficacy.

meet the regulatory requirements (US FDA, 1998). The US Food and Drug Administration Guidance on a specific dry powder product (combined fluticasone propionate and salmeterol xinofoate) dry powder inhaler intended to lead to in vitro and in vivo bioequivalence (Apiou-Sbirlea G. et al., 2013) serves to emphasize the importance of understanding and controlling all phenomena described above (US FDA 2013). Greater progress is required in linking the spectrum of known properties to defined process variables if sufficient knowledge and understanding is to be gained to predict and efficiently control performance.

\section{Conclusion}

The complexity of powder behavior in DPIs can be considered at several levels. The first is the composition and structure of individual particles. The second is the composition and structure of populations of particles at rest. The third is the motion of particles as a partially dilated flowing mass. The fourth is the motion of particles as fully dilated dispersed aerosols. Each of these elements is intricately woven into the overall performance of the product dictating the delivered dose and its aerodynamic particle size distribution. These performance measures in conjunction with pulmonary function, anatomy and phys- 
olnug.

Acknowledging that complexity at all levels of scrutiny in powder properties and behavior emphasizes the difficulty of attempting to control static variables and expecting controlled product performance. A comprehensive understanding of the dynamics is central to managing performance within acceptable limits.

\section{References}

Adams W.P., Lee S.L., Plourde R., Lionberger R.A., Bertha C.M., Doub W.H., Bovet J.-M., Hickey A.J., Effects of device and formulation on in vitro performance of dry powder inhalers, The AAPS Journal, 14 (2012) 400-409.

Allen T.A., Microscopy, in: Particle Size Measurement, fourth ed., Chapman and Hall, New York, 1990, pp. 217-248.

Apiou-Sbirlea G., Newman S., Fleming J., Siekmeier R., Ehrmann S., Scheuch G., Hochhaus G., Hickey A., Bioequivalence of inhaled drugs: Fundamentals, challenges and perspectives, Therapeutic Delivery, 4 (2013) 343-367.

Atkins P.J., Crowder T.M., The design and development of inhalation drug delivery systems, in: Pharmaceutical Inhalation Aerosol Technology, second ed., Hickey A.J. (Ed.), Informa Healthcare, New York, 2004, pp. 279-309.

Begat P., Morton D.A.V., Staniforth J.N., Price R., The cohesiveadhesive balances in dry powder inhaler formulations I: Direct quantification by atomic force microscopy, Pharmaceutical Research, 21 (2004) 1591-1597.

Behara S.R.B., Larson I., Kippax P., Stewart P., Morton D.A.V., Insight into pressure drop dependent efficiencies of dry powder inhalers, European Journal of Pharmaceutical Sciences, 46 (2012) 142-148.

Behara S.R.B., Kippax P., McIntosh M.P., Morton D.A.V., Larson I., Stewart P., Structural influence of cohesive mixtures of salbutamol sulphate and lactose on aerosolisation and de-agglomeration behaviour under dynamic conditions, European Journal of Pharmaceutical Sciences, 42 (2011a) 210-219.

Behara S.R.B., Larson I., Kippax P., Morton D.A.V., Stewart P., An approach to characterising the cohesive behaviour of powders using a flow titration aerosolisation based methodology, Chemical Engineering Science, 66 (2011b) 16401648 .

Behara S.R.B., Larson I., Kippax P., Morton D.A.V., Stewart P., The kinetics of cohesive powder de-agglomeration from three inhaler devices, International Journal of Pharmaceutics, 421 (2011c) 72-81.

Behara S.R.B., Kippax P., Larson I., Morton D.A.V., Stewart P., Kinetics of emitted mass - a study with three dry powder inhaler devices, Chemical Engineering Science, 66 (2011d) 5284-5292.

Carstensen J., Solid state stability, in: McGinity J.W. (Ed.), Drug Stability: Principles and Practices, Marcel Dekker, Inc., New York, 1990, pp. 129-164

Carstensen J.T., Angle of repose, in: Carstensen J.T. (Ed.) Phar- maceutical Principles of Solid Dosage Forms, Technomic Publishing Co, Inc., Lancaster, 1993, pp. 27-29.

Chew N.Y.K., Chan H.-K., Use of solid corrugated particles to enhance powder aerosol performance, Pharmaceutical Research, 18 (2001) 1570-1577.

Concessio N.M., VanOort M.M., Knowles M.R., Hickey A.J., Pharmaceutical dry powder aerosols: Correlation of powder properties with dose delivery and implications for pharmacodynamic effect, Pharmaceutical Research, 16 (1999) 828-834.

Crowder T.M., Hickey A.J., An instrument for rapid powder flow measurement and temporal fractal analysis, Particle \& Particle Systems Characterization, 16 (1999) 32-34.

Crowder T.M., Hickey A.J., The physics of powder flow: Applied to pharmaceutical solids, Pharmaceutical technology., 24 (2000) 50-58.

Crowder T.M., Hickey A.J., Louey M.D., Orr N., Particulate systems: manufacture and characterization, Interpharm/ CRC, Boca Raton, FL, 2003, pp. 9-26.

Crowder T., Hickey A., Powder specific active dispersion for generation of pharmaceutical aerosols, International Journal of Pharmaceutics, 327 (2006) 65-72.

Crowder T.M., Rosati J.A., Schroeter J.D., Hickey A.J., Martonen T.B., Fundamental effects of particle morphology on lung delivery: Predictions of stokes' law and the particular relevance to dry powder inhaler formulation and development, Pharmaceutical Research, 19 (2002) 239-245.

Crowder T.M., Sethuraman V., Fields T.B., Hickey A.J., Signal processing and analysis applied to powder behavior in a rotating drum, Particle \& Particle Systems Characterization, 16 (1999) 191-196.

Davies C.N., Particle fluid interactions, J. Aerosol Sci., 10 (1979) 477-513.

Dunbar C.A., Hickey A.J., Holzner P., Dispersion and characterization of pharmaceutical dry powder aerosols, KONA Powder and Particle Journal, 16 (1998) 7-45.

Dunbar C.A., Morgan B., Van Oort M., Hickey A.J., comparison of dry powder inhaler dose delivery characteristics using a power criterion, PDA Journal of Pharmaceutical Science and Technology, 54 (2000) 478-484.

Fults K.A., Miller I.F., Hickey A.J., Effect of particle morphology on emitted dose of fatty acid-treated disodium cromoglycate powder aerosols, Pharmaceutical Development and Technology, 2 (1997) 67-79.

Gonda I., el Khalik A.F.A., Aerodynamic properties of different crystal forms of hexamethylmelamine, The Annals of Occupational Hygiene, 32 (1988) 379-390.

Grasmeijer F., Hagedoorn P., Frijlink H.W., de Boer A.H., Drug content effects on the dispersion performance of adhesive mixtures for inhalation, PLoS ONE, 8 (2013) e71339.

Grasmeijer F., Lexmond A.J., Maarten V., Hagedoorn P., Hickey A.J., Frijlink H.W., de Boer A.H., New mechanisms to explain the effects of added lactose fines on the dispersion performance of adhesive mixtures for inhalation, PLoS One, 9 (2014) 1-11.

Hickey A.J., Lung deposition and clearance of pharmaceutical aerosols: What can be learned from inhalation toxicology and industrial hygiene?, Aerosol Science and Technology, 
r.

Ne 18 .(P993) 290-304.

Hickey A.J., Pharmaceutical inhalation aerosol powder dispersion-An unbalancing act, American Pharmaceutical Review, 6 (2003) 106-110.

Hickey A.J., Summary of common approaches to pharmaceutical aerosol administration, in: Pharmaceutical Inhalation Aerosol Technology, second ed., A.J. Hickey (Ed.), Marcel Dekker, Inc., New York, NY, 2004, pp. 385-421.

Hickey A.J., Concessio N.M., Flow properties of selected pharmaceutical powders from a vibrating spatula, Particle \& Particle Systems Characterization, 11 (1994) 457-462.

Hickey A.J., Concessio N.M., Chaos in rotating lactose powder beds, Particulate Science and Technology, 14 (1996) 15-25.

Hickey A.J., Concessio N.M., Descriptors of irregular particle morphology and powder properties, Advanced Drug Delivery Reviews, 26 (1997) 29-40.

Hickey A.J., Ganderton D. (Eds.), Size reduction and classification, in: Pharmaceutical Process Engineering, Informa Healthcare, New York, 2001, pp. 174-197.

Hickey A.J., Mansour H.M., Telko M.J., Xu Z., Smyth H.D.C., Mulder T., McLean R., Langridge J., Papadopoulos D., Physical characterization of component particles included in dry powder inhalers. I. Strategy review and static characteristics, J Pharm Sci, 96 (2007a) 1282-1301.

Hickey A.J., Mansour H.M., Telko M.J., Xu Z., Smyth H.D.C., Mulder T., McLean R., Langridge J., Papadopoulos D., Physical characterization of component particles included in dry powder inhalers. II. Dynamic characteristics, J Pharm Sci, 96 (2007b) 1302-1319.

Hickey A.J., Xu Z., Balancing particle properties to achieve effective inhaled drug delivery, Pharm Tech Japan, 29 (2013) 183-188.

Hinds W.C., Aerosol technology: Properties and Behavior and Measurement of Airborne Particles, second ed., John Wiley and Sons, New York, 1999, p. 251.

Jaffari S., Forbes B., Collins E., Barlow D.J., Martin G.P., Murnane D., Rapid characterisation of the inherent dispersibility of respirable powders using dry dispersion laser diffraction, International Journal of Pharmaceutics, 447 (2013) 124-131.

Jaffari S., Forbes B., Collins E., Khoo J., Martin G.P., Murnane D., Evidence for the existence of powder sub-populations in micronized materials: Aerodynamic size-fractions of aerosolized powders possess distinct physicochemical properties, Pharmaceutical Research, 31 (2014) 3251-3264.

Jones M.D., Price R., The influence of fine excipient particles on the performance of carrier-based dry powder inhalation formulations, Pharmaceutical Research, 23 (2006) 16651674.

Lippmann M., Effects of fiber characteristics on lung deposition, retention, and disease, Environmental health perspectives, 88 (1990) 311-317.

Louey M.D., Van Oort M., Hickey A.J., Standardized entrainment tubes for the evaluation of pharmaceutical dry powder dispersion, Journal of Aerosol Science, 37 (2006) 15201531.

Mansour H.M., Xu Z., Hickey A.J., Dry powder aerosols generated by standardized entrainment tubes from alternative sugar blends: 3 . Trehalose dihydrate and D-mannitol carriers, Journal of Pharmaceutical Sciences, 99 (2010) 34303441 .

Martonen T.B., Katz I., Fults K., Hickey A.J., Use of analytically defined estimates of aerosol respirable fraction to predict lung deposition patterns, Pharmaceutical Research, 9 (1992) 1634-1639.

Mullin J.W., Crystallization, third ed., Butterworth-Heinemann, Oxford, 1993.

Sanders M., Pulmonary drug delivery: An historical overview, in: Smyth H.D.C., Hickey A.J. (Eds.), Controlled pulmonary drug delivery, Springer New York, New York, NY (2011) pp. 51-73.

Shah I., The blind ones and the matter of the elephant, in: Tales of the Dervishes, Arkana, London, 1993, pp. 25-26.

Shur J., Kubavat H.A., Ruecroft G., Hipkiss D., Price R., Influence of crystal form of ipratropium bromide on micronisation and aerosolisation behaviour in dry powder inhaler formulations, Journal of Pharmacy and Pharmacology, 64 (2012) 1326-1336.

Smyth H.D.C., Cooney D.J., Garmise R.J., Zimmerer R.O., Pipkin J.D., Hickey A.J., Dynamic electrostatic charge determinations of dry powder blends for inhalation: correlations with dispersion performance influence of physical form of captisol, in: Respiratory Drug Delivery IX, Volume III, Dalby R.N., Byron P.R., Peart J., Suman J.D. and Farr S.J. (Eds.), Davis Healthcare International Publishing, LLC, River Grove, IL, 2004, pp. 805-807.

Steckel H., Bolzen N., Alternative sugars as potential carriers for dry powder inhalations, International Journal of Pharmaceutics, 270 (2004) 297-306.

Stewart P.J., Particle interaction in pharmaceutical systems, Pharmaceutical International, 7 (1986) 146-149.

Taylor M.K., Ginsburg J., Hickey A.J., Gheyas F., Composite method to quantify powder flow as a screening method in early tablet or capsule formulation development, AAPS PharmSciTech, 1 (2000) 20-30.

Telko M.J., Hickey A.J., Dry powder inhaler formulation, Respiratory Care, 50 (2005) 1209-1227.

Telko M.J., Hickey A.J., Critical assessment of inverse gas chromatography as means of assessing surface free energy and acid-base interaction of pharmaceutical powders, Journal of Pharmaceutical Sciences, 96 (2007) 2647-2654.

Telko M.J., Hickey A.J., Aerodynamic and electrostatic properties of model dry powder aerosols: A comprehensive study of formulation factors, AAPS PharmSciTech, 15 (2014) 1378-1397.

Telko M.J., Kujanpää J., Hickey A.J., Investigation of triboelectric charging in dry powder inhalers using electrical low pressure impactor (ELPITM), International Journal of Pharmaceutics, 336 (2007) 352-360.

Tian Y., Parasrampuria D., Kanke M., Hickey A.J., Manufacture and in-vitro assessment of bromsulphthalein and phenolphthalein loaded polyester microspheres, Drug Development and Industrial Pharmacy, 20 (1994) 1159-1178.

Tougas T.P., Mitchell J.P., Lyapustina S.A., Good Casacade Impactor Practices, AIM and EDA for Orally Inhaled Products, Springer, NY, 2013. 
LS FDA (Food and Drug Adminstration), November 1998

WDraft Guidance for the Industry, Metered Dose inhaler (MDI and Dry Powder Inhaler (DPI) Chemistry, Manufacturing and Controls Documentation.

US FDA (Food and Drug Administration), Draft Guidance on Fluticasone Propionate; Salmeterol Xinafoate, September 2013.

USP (United States Pharmacopoeia), General Chapter $<601>$ Aerosols, Nasal Sprays, Metered Dose Inhalers, and Dry Powder Inhalers, USP Rockville, MD, 34 (2011) pp. 218239.

Van Oort M.M., Sacchetti M., Spray drying and supercritical fluid particle generation techniques, in: Inhalation Aerosols, Physical and Biological Basis for Therapy, Second Edition, A.J. Hickey (Eds.), Informa Healthcare, New York, 2007, pp. 307-346.

Vehring R., Pharmaceutical particle engineering via spray drying, Pharmaceutical Research, 25 (2008) 999-1022.

Wong J., Lin Y.-W., Kwok P.C.L., Niemelä V., Crapper J., Chan H.-K., Measuring bipolar charge and mass distributions of powder aerosols by a novel tool (BOLAR)., Molecular Pharmaceutics, 12 (2015) 3433-3440.

$\mathrm{Xu}$ Z., Mansour H.M., Hickey A.J., Particle interactions in dry powder inhaler unit processes: A review, Journal of Adhesion Science and Technology, 25 (2011) 451-482.

Xu Z., Mansour H.M., Mulder T., McLean R., Langridge J., Hickey A.J., Dry powder aerosols generated by standardized entrainment tubes from drug blends with lactose monohydrate: 1. Albuterol sulfate and disodium cromogly- cate, Journal of Pharmaceutical Sciences, 99 (2010a) 33983414.

Xu Z., Mansour H.M., Mulder T., McLean R., Langridge J., Hickey A.J., Dry powder aerosols generated by standardized entrainment tubes from drug blends with lactose monohydrate: 2 . Ipratropium bromide monohydrate and fluticasone propionate, Journal of Pharmaceutical Sciences, 99 (2010b) 3415-3429.

Xu Z., Mansour H.M., Mulder T., McLean R., Langridge J., Hickey A.J., Heterogeneous particle deaggregation and its implication for therapeutic aerosol performance, Journal of Pharmaceutical Sciences, 99 (2010c) 3442-3461.

Young P.M., Chan H.-k., Chiou H., Edge S., Tee T.H.S., Traini D., The influence of mechanical processing of dry powder inhaler carriers on drug aerosolization performance, Journal of Pharmaceutical Sciences, 96 (2007) 1331-1341.

Young P.M., Cocconi D., Colombo P., Bettini R., Price R., Steele D.F., Tobyn M.J., Characterization of a surface modified dry powder inhalation carrier prepared by "particle smoothing", Journal of Pharmacy and Pharmacology, 54 (2002) 1339-1344.

Zhou Q., Qu L., Gengenbach T., Larson I., Stewart P.J., Morton D.A.V., Effect of surface coating with magnesium stearate via mechanical dry powder coating approach on the aerosol performance of micronized drug powders from dry powder inhalers, AAPS PharmSciTech, 14 (2013) 38-44.

Ziffels S., Bemelmans N.L., Durham P.G., Hickey A.J., In vitro dry powder inhaler formulation performance considerations, Journal of Controlled Release, 199 (2015) 45-52.

\section{Author's short biography}

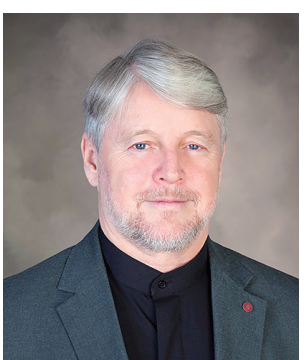

Anthony J. Hickey

Dr. Hickey is Distinguished RTI Fellow, at RTI International, Emeritus Professor of Molecular Pharmaceutics of the Eshelman School of Pharmacy, and Adjunct Professor Biomedical Engineering in the School of Medicine, at the University of North Carolina at Chapel Hill. He obtained Ph.D. and D.Sc. degrees in pharmaceutical sciences from Aston University, Birmingham, UK. He is a Fellow of the Royal Society of Biology, the American Association of Pharmaceutical Scientists and the American Association for the Advancement of Science. He received the Research Achievement Award of the Particulate Presentations and Design Division of the Powder Technology Society of Japan and the David W Grant Award in Physical Pharmacy of the American Association of Pharmaceutical Scientists. He is a member of the Pharmaceutical Dosage Forms Expert Committee of the United States Pharmacopeia. 